\title{
A Novel Technique - Bone Splitting and Bone Grafting in an Hourglass-shaped Bone Following Distraction Osteogenesis
}

\author{
Huang S Lee ${ }^{1}$, Rohaman Tasarib² ${ }^{2}$ Fahrudin $\mathrm{CHamzah}^{3}$, Ashraf HA Halim
}

\begin{abstract}
Aim: We describe a novel technique of bone splitting and bone grafting in managing hypoplastic or hourglass-shaped regenerated bone in distraction osteogenesis.

Background: Hourglass-shaped regenerated bone is a potential complication during distraction osteogenesis which is vulnerable to fracture when loaded. Our novel technique overcomes this by increasing the diameter of new bone formation via bone splitting and bone grafting.

Case description: We report three cases with hypoplastic regenerated bone following distraction osteogenesis. It was treated with bone splitting and bone grafting. Although one case was complicated with an iatrogenic transverse fracture during the surgery, all three cases achieved the goal of increasing bone diameter during the subsequent consolidation phase.

Conclusion: This relatively simple and novel surgical intervention can overcome the hourglass-shaped appearance, thus preventing potential fracture.

Clinical significance: We emphasise the importance of identifying hypoplastic regenerate bone before the consolidation phase of distraction osteogenesis. The novel technique described is a simple surgical intervention which can prevent potential fracture through the newly formed bone.

Keywords: Distraction osteogenesis, Hourglass, Hypoplastic, Ilizarov.

Strategies in Trauma and Limb Reconstruction (2020): 10.5005/jp-journals-10080-1509
\end{abstract}

\section{INTRODUCTION}

Ilizarov bone transport is a widely used method among orthopaedic surgeons in managing long bone defects. However, it has its own potential shortcomings. One of the problems at the distraction site is the potential formation of hourglass-shaped regenerated bone which has a smaller diameter than the native bone. This has the potential to fracture when loaded. Currently, there is no treatment protocol for such problem. We report a case series of novel bone splitting technique and bone grafting to increase the diameter of the hourglass - shaped bones at the distraction site. To our best knowledge, such a method has not been described in the literature.

\section{Case Description}

\section{Case 1}

A 21-year-old lady sustained an open fracture of the left femur which was treated with a locking compression plate (LCP) after adequate debridement. It was complicated with an infected non-union (Fig. 1A). We proceeded with removal of the LCP, bone resection, and acute docking of the left femur with gradual bone lengthening using a monorail external fixator from proximal to distal (Fig. 1B). During follow-up, serial radiographs showed good osteogenesis and bone union at the docking site. However, the newly formed bone at the distraction site was smaller in diameter with an hourglass appearance in both coronal and sagittal planes (Fig. 1C).

We proceeded with bone splitting and bone grafting. A longitudinal skin incision was made at the narrowest part of the bone and on the periosteum (Fig. 4). The bone was adequately exposed with two bone elevators (Fig. 5A). Using an osteotome, two perpendicular planes of osteotomy were made on the hourglassshaped bone. The osteotome was used to hinge-open the split 1,3,4 Orthopaedic Department, University Putra Malaysia, Serdang,
Selangor, Malaysia
Orthopaedic Department, Hospital Serdang, Kajang, Selangor,
Malaysia

Corresponding Author: Huang S Lee, Orthopaedic Department, University Putra Malaysia, Serdang, Selangor, Malaysia, Phone: +6012 3662398, e-mail: jeffrey_leehs@yahoo.com

How to cite this article: Lee HS, Tasarib R, Hamzah FC, et al. A Novel Technique-Bone Splitting and Bone Grafting in an Hourglass-shaped Bone Following Distraction Osteogenesis. Strategies Trauma Limb Reconstr 2020;15(3):175-178.

Source of support: Nil

Conflict of interest: None

bone creating a gap and making the diameter of the bone larger (Fig. 1D). The space was filled with demineralised bone matrix (DBM), which acts as a strut to increase and maintain the bone diameter (Fig. 5B). At 2 months post-splitting, the osteotomy line was still visible (Fig. 1E). The gap was completely obliterated at 6 months postoperation with an increased bone diameter as compared to before. The monorail fixator was subsequently removed (Fig. 1F).

\section{Case 2}

A 50 year-old gentleman sustained a grade 3 a open fracture of the right tibia and fibula. He underwent debridement and removal of a large segment of devitalised bone. Initial stabilisation was secured with an external fixator. (Fig. 2A). This was subsequently exchanged with an llizarov external fixation (IEF) for bone transport (Fig. 2B). The bone transport was complicated with an hourglass-shaped bone despite the patient being compliant 

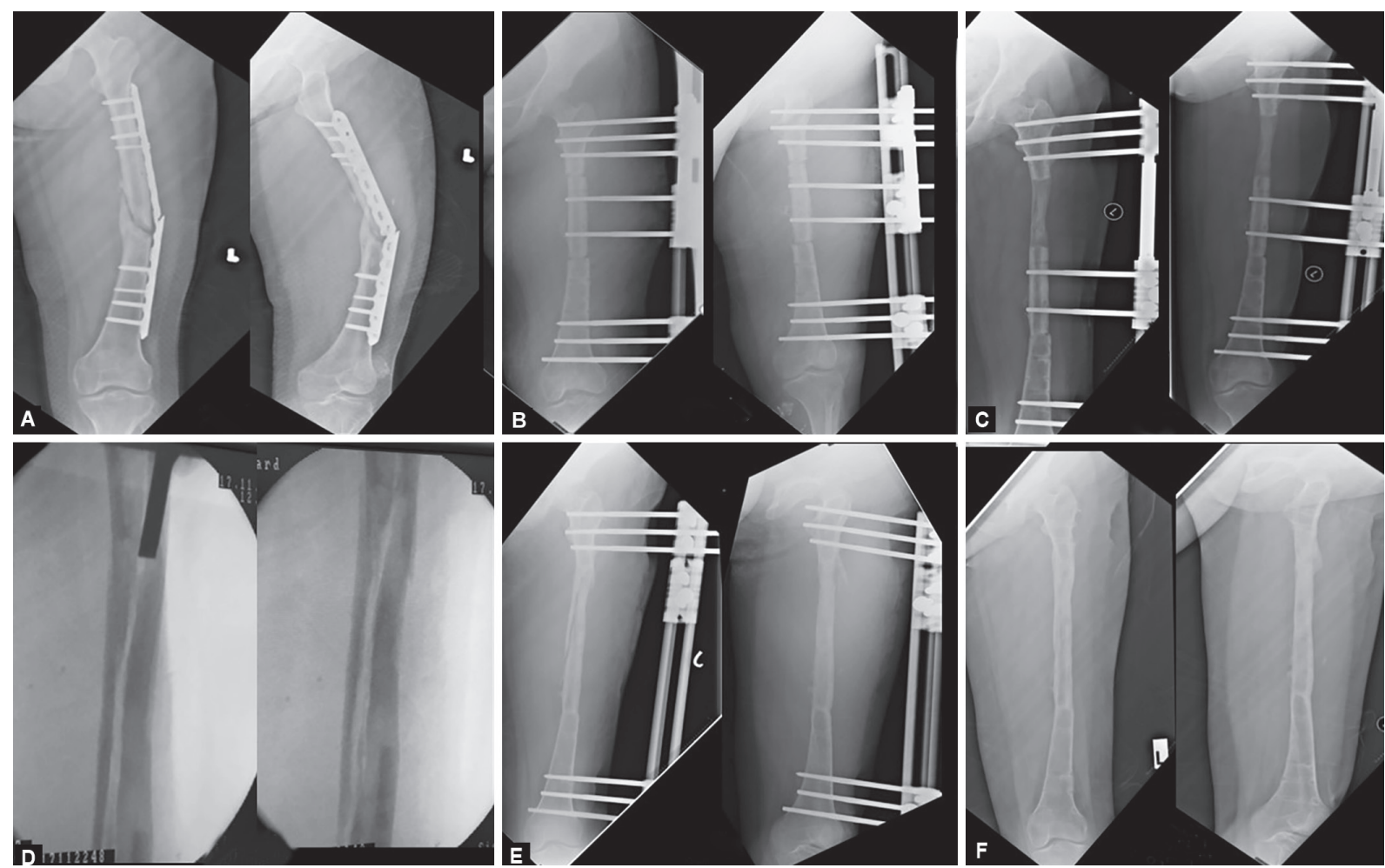

Figs. $1 \mathrm{~A}$ to F: Case 1: Anteroposterior and lateral view X-rays showing (A) Non-union with broken implant; (B) Bone resection and acute docking; (C) Hourglass appearance bone formation at distraction site; (D) Intraoperative bone splitting images; (E) Line still visible at 2 months after bone splitting; (F) Six-month post-operation with increased bone diameter and consolidation
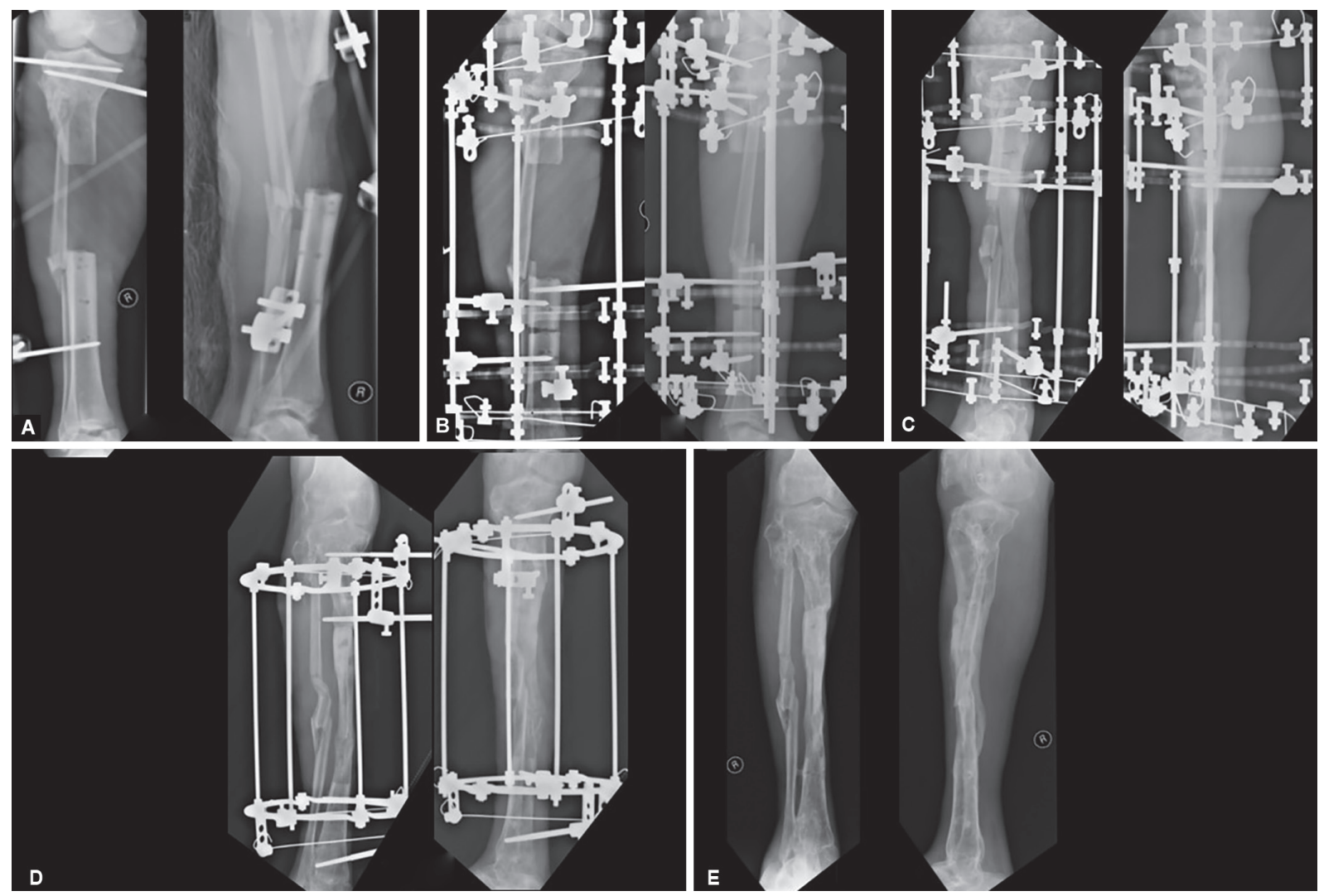

Figs. 2A to E: Case 2: Anteroposterior and lateral view X-rays showing (A)Bone resection during initial debridement; (B) Bone resection and IEF application; (C) Hourglass appearance at distraction site; (D) Osteotomy line visible at 2 months postoperation; (E) Six months after bone splitting with bone union 

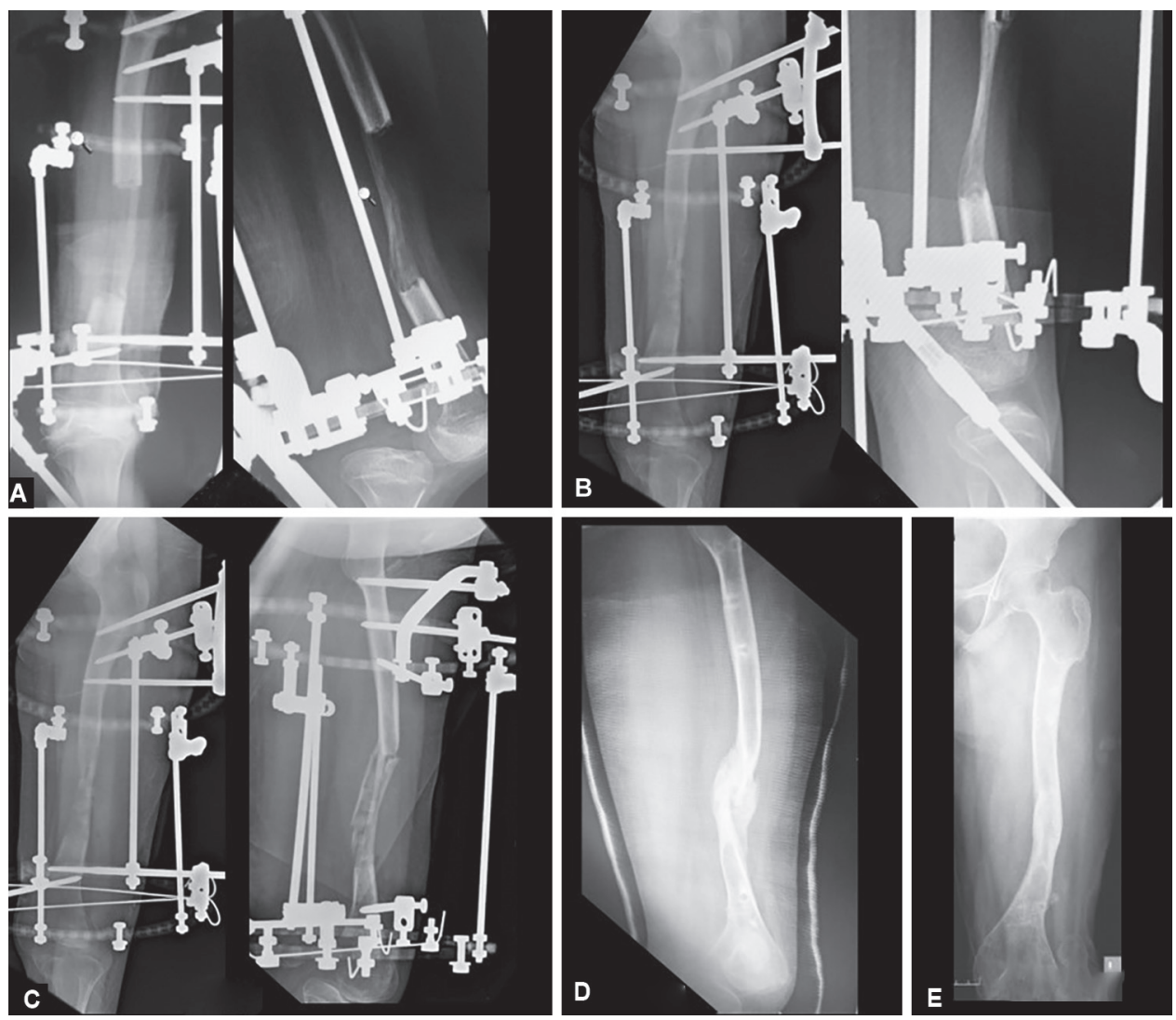

Figs. 3A to E: Case 3: Anteroposterior and lateral view X-rays showing (A) Early sign of hourglass appearance; (B) Persistent hourglass appearance despite full weight-bearing; (C) After bone splitting, complicated with complete fracture; (D) Healing fracture site with increased bone diameter; (E) Remodelled left femur after 6 months of bone splitting and bone graft.

with weight-bearing 6 months postoperation (Fig. 2C). The bone splitting and DBM bone grafting technique used was similar to the first case. The bone splitting line was still visible at 2 months post-operation but was not visible at 6 months post-operation. There was an increase in bone diameter as compared to before (Figs 2D and E). The IEF was taken off at that point.

\section{Case 3}

A 14-year-old female with a congenital longitudinal deficiency of the left femur underwent a bone-lengthening procedure. It was complicated with a dislocated knee during the course of lengthening and an hourglass appearance at the distraction site (Fig. 3A). Fortunately, there was no neurovascular function compromise and the dislocation was addressed successfully by gradual reduction using a hexapod device (Ortho-SUV) with some residual limited range of movement $\left(5-100^{\circ}\right)$ (Fig. 3B). The regenerate bone column was treated by bone splitting and DBM bone grafting similar to the previous two cases but developed a complete transverse fracture intraoperatively (Fig. 3C). Serial follow-up showed healing of the fracture site and remodelled larger diameter of the left femur at 6 months postsurgery (Figs 3D and E).

\section{Discussion}

Bone transport and bone lengthening via distraction osteogenesis (DO) is gaining popularity among the orthopaedic surgeons in managing bone defects and limb shortening. There are a few recognised potential complications, such as pin-site infections,

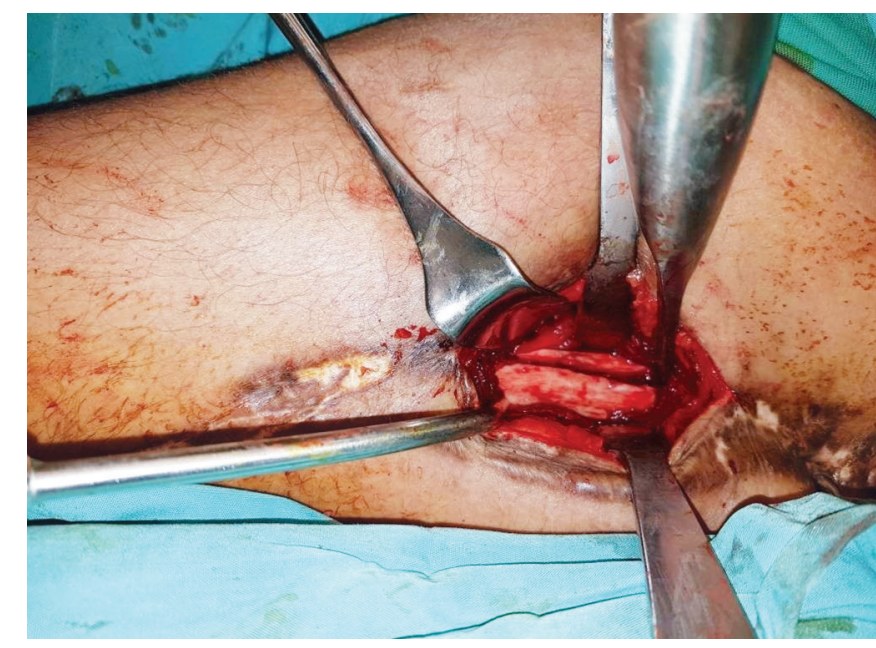

Fig. 4: It shows the longitudinal incision made over the level of interest. The bone segment was exposed and two perpendicular planes of osteotomy were carried out

muscle contractures, neurovascular injuries, and joint subluxations. An hourglass appearance of the regenerate bone can occur in up to $13.8 \%$ of the cases. ${ }^{1}$ This problem is challenging to manage and has not, to our best knowledge, been reported widely in the literature and is without standard treatment. 

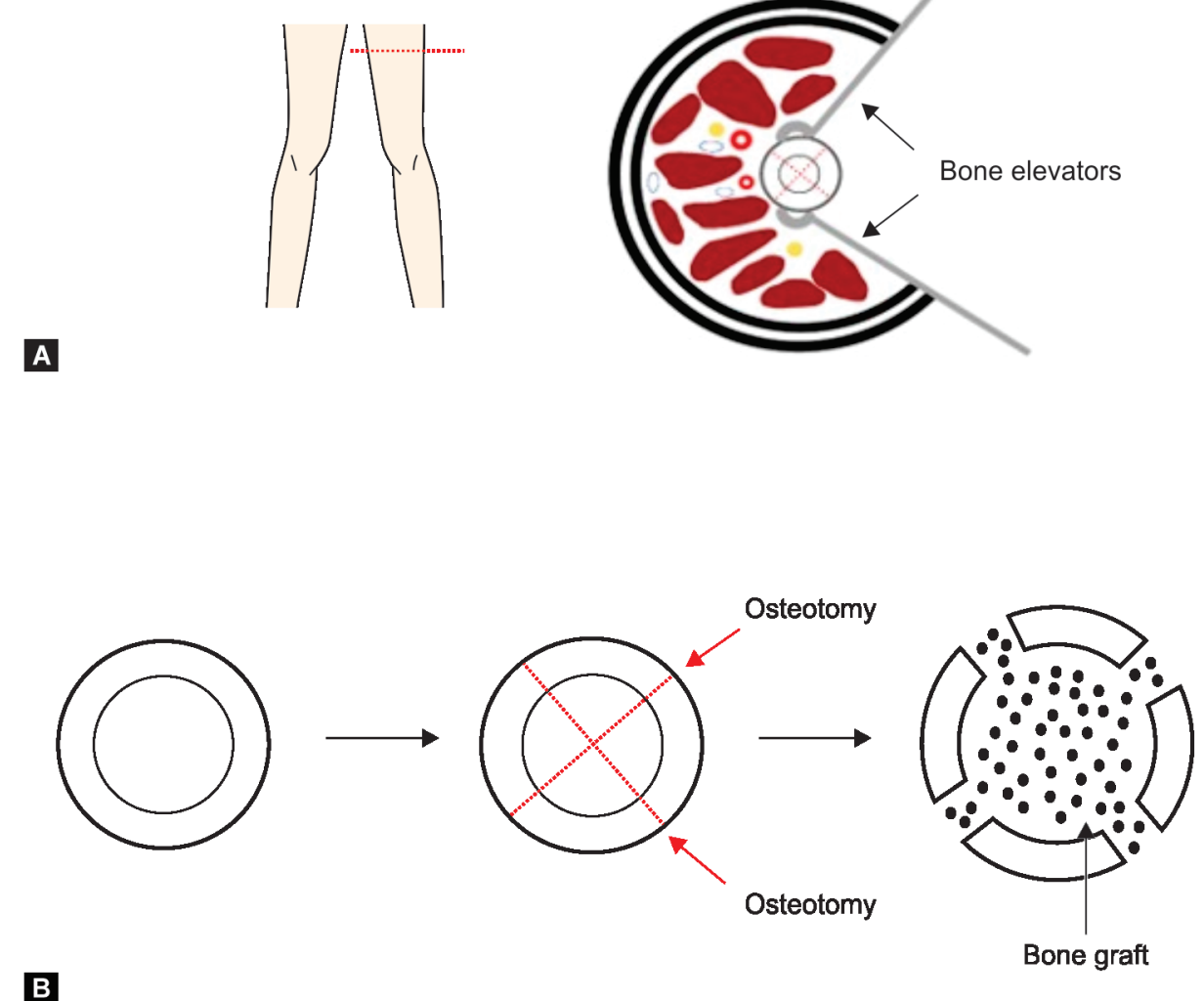

Figs. 5A and B: (A) It illustrates the cross-section of the left thigh with bone elevators exposing the femur; (B) It illustrates the two perpendicular planes of osteotomy directions. The bone graft was then inserted to increase the femur diameter

The initial osteotomy for DO was performed in the diaphyseal region which carries a smaller transport segment diameter. We postulate that this could have led to the hourglass-shaped regenerate bone and that such osteotomies for transport or lengthening should be performed preferably at the wide metaphyseal region to avoid such complications. Li et al. classify the hourglass appearance as shape 3 and note that such regenerate formation is associated with a poor bony response to lengthening or an over-vigorous distraction rate. ${ }^{2}$ The periosteum, thought to be important for bone regeneration, may have contributed to the hourglass shape due to the lack of extension of the periosteum. ${ }^{3}$ Therefore, it is important to preserve the periosteum with the utmost care during osteotomy or bone splitting procedures. Frame instability, vascular insufficiency, and lack of function or weightbearing are the other possible reasons. ${ }^{4}$

The hourglass-shaped bone is less sturdy and is vulnerable to fracture once loaded. ${ }^{5}$ To avoid this complication, especially in weight-bearing bones, we propose that a preventive surgery should be carried out before the consolidation phase. The aim is to widen the diameter of the newly formed bone to be almost equal to the native bone, hence decreasing the likelihood of a fracture. This novel technique was inspired by a local dish known as bamboo rice whereby the only way to remove the cooked rice from inside the bamboo is to enlarge the bamboo's diameter by splitting it. We used this concept by applying two longitudinal split planes perpendicular to each other. The gap is then filled with either synthetic or autologous bone graft. We chose DBM as a
Table 1: Progress for each case in relation to bone splitting

\begin{tabular}{|c|c|c|c|}
\hline & $\begin{array}{l}\text { Distraction } \\
\text { duration, } \\
\text { weeks }\end{array}$ & $\begin{array}{l}\text { Time from } \\
\text { completion of } \\
\text { distraction to } \\
\text { bone splitting, } \\
\text { weeks }\end{array}$ & $\begin{array}{l}\text { Time from } \\
\text { bone } \\
\text { splitting to } \\
\text { remodelling, } \\
\text { weeks }\end{array}$ \\
\hline \multicolumn{4}{|l|}{ Case 1} \\
\hline Bone lengthening: $7 \mathrm{~cm}$ & 9 & 20 & 22 \\
\hline \multicolumn{4}{|l|}{ Case 2} \\
\hline Bone transport: $4 \mathrm{~cm}$ & 6 & 14 & 21 \\
\hline \multicolumn{4}{|l|}{ Case 3} \\
\hline Bone lengthening: $6 \mathrm{~cm}$ & 8 & 18 & 21 \\
\hline
\end{tabular}

graft material because our patients were not keen on the potential donor site morbidity, such as prolonged pain, hypersensitivity, or meralgia paraesthetica. In using synthetic bone graft to assist in bone healing, we are aware that this may have taken longer to do so than with an autologous bone graft. This could be due to the lack of osteogenic properties in the synthetic bone graft. Table 1 shows that with synthetic bone graft it took approximately 21 to 22 weeks for all the bones to unite and remodel regardless of their length. Our experience also showed that even with an iatrogenic complete fracture during bone splitting as occurred in case 3 , the bone heals while achieving our objective of increasing its diameter. 


\section{Conclusion}

In conclusion, we advocate performing a relatively simple procedure to increase the diameter of a newly formed hourglassshaped bone following DO to prevent a possible future fracture. In our case series, we have shown that the novel technique of bone splitting and bone grafting achieved this objective successfully.

\section{Clinical Significance}

We emphasise the importance of identifying a hypoplastic regenerate bone before the consolidation phase of $\mathrm{DO}$. The novel technique described is a simple surgical intervention which can prevent potential fracture through the newly formed bone.

\section{References}

1. Borzunov D, Shastov A. Ischemic distraction regenerate: interpretation, definition, problems and solutions. Traumatol Orthop Russia 2019 March;25(1):68-76. Available from:
https://www.researchgate.net/publication/333143252_Ischemic Distraction_Regenerate_Interpretation_Definition_Problems_ and_Solutions. DOI: 10.21823/2311-2905-2019-25-1-68-76.

2. Li R, Yang L. Radiographic classification of osteogenesis during bone distraction. J Orthop Res 2006 March;24(3):339-347. Available from: https://www.researchgate.net/publication/7296555_Radiographic classification_of_osteogenesis_during_bone_distraction. DOI: 10.1002/jor.20026.

3. Hamada M, Sakamoto Y, Nagasao T, et al. Treatment of complications after distraction osteogenesis for brachymetatarsia of the fourth metatarsal. Plant Reconstr Surg Glob Open 2016 July;4;e817. Available from: https://www.ncbi.nlm.nih.gov/pmc/articles/PMC4977145/. DOI: $10.1097 /$ GOX.0000000000000836.

4. Khaleel A, Pool RD. Bone transport. Curr Orthop 2001 June;5(3):229-237. Available from: https://www.researchgate.net/ publication/246836597_Bone_transport.DOI: 10.1054/cuor.2001.0173.

5. Lamm BM, Gourdine-Shaw MC. Problems, obstacles, and complications of metatarsal lengthening for the treatment of brachymetatarsia. Clin Podiatr Med Surg 2010 October;27(4):561-582. Available from: https:// www.researchgate.net/publication/47383307_Problems_Obstacles_ and_Complications_of_Metatarsal_Lengthening_for_the Treatment_of_Brachymetatarsia. DOI: 10.1016/j.cpm.2010.06.006. 